\title{
Hacia una complexión del discurso intercultural en planes de estudio de educación básica
}

Understanding Intercultural Discourse in the Curriculum of Mexico's Basic Education

\author{
Volumen 19, Número 1 \\ Enero-Abril \\ pp. $1-28$
}

\section{Este número se publica el 1 de enero de 2019}

DOI 10.15517/aie.v19i1.35255

Sergio Gerardo Malaga-Villegas

Revista indizada en REDALYC, $\underline{\text { SCIELO }}$

Revista distribuida en las bases de datos:

LATINDEX, DOAJ, REDIB, IRESIE, CLASE, DIALNET, SHERPA/ROMEO, QUALIS-CAPES, MIAR

Revista registrada en los directorios:

ULRICH'S $, \underline{R E D I E}, \underline{R I N A C E}, \underline{\text { OEI }}, \underline{\text { MAESTROTECA }}, \underline{\text { PREAL, }} \underline{\text { CLACSO }}$ 


\section{Hacia una complexión del discurso intercultural en planes de estudio de educación básica

\author{
Understanding Intercultural Discourse in the Curriculum of Mexico's Basic Education
}

Sergio Gerardo Malaga-Villegas ${ }^{1}$

Resumen: En este ensayo se analizan las significaciones del discurso intercultural en los planes de estudio de educación básica. Se privilegiaron los planes publicados en lo que va de este siglo (preescolar 2004, secundaria 2006, primaria 2009, plan de educación básica 2011 y Modelo Educativo para la Educación Obligatoria 2017). A la constelación resultante de sentidos y significaciones se le denominó complexión escolarizada del discurso intercultural. El principal argumento que se sostiene es que el imaginario de la Secretaría de Educación Pública, condensado en esos documentos normativos, tiende a ser reducido y exclusionista respecto de la producción de conocimiento de la educación intercultural gestada a nivel nacional. En términos organizativos, se presenta un breve estado de conocimiento, se continúa con el análisis de la complexión escolarizada en los planes de estudio arriba señalados, y se cierra con algunas reflexiones finales.

Palabras clave: educación intercultural, plan de estudios, educación básica, México.

Abstract: This essay analyzes intercultural discourse in the curriculum of Mexico's basic education. Focuses on the curricula published in this century (preschool 2004, secondary school 2006, elementary school 2009, Integrated Reform of Basic Education 2011, and Educational Model for Basic Education 2017). This constellation of meanings was referred as the institutionalized intercultural discourse. The main argument is that the ideal of Mexico's Secretariat of Public Education, as condensed in these curricula, tend to be reduced and exclusionist with respect to the field of knowledge of intercultural education at the national level. In organizational terms, this essay is located within the framework of a brief state of knowledge, following with the analysis of intercultural discourse in the indicated curricula, and it concludes with suggestions for continuing the discussion.

Key Words: intercultural education, curriculum, basic education, Mexico.

\footnotetext{
1 Investigador de Tiempo Completo Titular "C" de la Universidad Autónoma de Baja California, Instituto de Investigación y Desarrollo Educativo, México. Doctor en Investigaciones Educativas, DIE-Cinvestav.
}

Dirección electrónica: smalaga@uabc.edu.mx

Ensayo recibido: 25 de junio, 2018

Enviado a corrección: 17 de octubre, 2018

Aprobado: 29 de octubre, 2018 


\section{Introducción}

El campo de la educación intercultural tal y como se conoce hasta ahora responde a una lógica compleja -multi, inter y transdisciplinar- respecto de las inquietudes de quienes investigan en, sobre y para dicho campo. Su configuración resulta inacabada e iterable (Derrida, 1989) debido al locus de enunciación (testimonial, documental o prescriptivo) y a los contextos (internacional, nacional o local) donde se producen tales conocimientos.

En este ensayo se analizan las significaciones del discurso intercultural. Para tal efecto, se eligieron los planes de estudio que la Secretaría de Educación Pública (SEP) renovó en lo que va de este siglo -preescolar 2004, secundaria 2006, primaria 2009, plan de educación básica 2011 y Modelo educativo para la educación obligatoria 2017- que, dicho sea de paso, se diseñaron en el marco de una Educación Intercultural para Todos. En razón de lo anterior, en este ensayo se privilegia el locus prescriptivo y el ámbito nacional.

Decantar por los planes mencionados responde a tres inquietudes. En principio, estipulan las orientaciones disciplinares, académicas y curriculares del quehacer educativo nacional, lo que incluye el tema de la educación intercultural. En segundo lugar, guardan un imaginario gubernamental que aspira a la formación ciudadana, actividad que, según la SEP, se desarrolla principalmente en los salones de clase. En tercer lugar, la difusión de dicho imaginario es llevada a cabo por quienes realizan docencia en educación básica, que, a su vez, juegan el rol de dispositivos de control del Estado (Althusser, 1988) y que son interpelados para asegurar una cierta transformación educativa del país (Fullan, 2002; Vaillant, 2005; Vezub, 2007).

El resultado de este análisis es una constelación discursiva, integrada por tres elementos: significantes, significaciones y sectores de atención de la educación intercultural. A esta constelación se le denominó complexión escolarizada por dos razones. Primeramente, porque la noción complexión al ser recuperada de la antropometría, y al instalarse en la arena educativa, ofreció la posibilidad de comprender los "matices epistemológicos [...] contextuales, discursivos, históricos” (Malaga-Villegas, 2012, p. 24) del discurso intercultural, ${ }^{2}$ característicos del locus y contexto en el que está inscrito este ensayo. En segundo lugar, porque el análisis de planes de estudio da cuenta de un tipo de

\footnotetext{
2 Si bien, en este ensayo se eligió la dimensión lingüística del discurso intercultural (planes de estudio), en ningún momento hay pretensión alguna de obviar su dimensión extralingüística (creación de instituciones, hechura de políticas educativas, puesta en marcha de programas educativos, etc.), la cual será tema de discusión en futuros trabajos.
} 
realidad escolarizada, la cual solo es posible comprenderse a partir del ideal gubernamental y de las finalidades de la SEP respecto de la educación intercultural y su relación con la formación ciudadana.

El argumento que se sostiene en este ensayo es que el discurso intercultural promovido por la SEP, tiende a ser reducido y exclusionista respecto de los avances que presenta el campo de conocimiento de la educación intercultural a nivel nacional. Ejemplo de este reduccionismo es que se ha privilegiado la valoración y el respeto de sectores indígenas, se visibiliza medianamente lo relacionado con la migración y se excluye a las otras diversidades (sociales, culturales, etc.) que constituyen un país multicultural como México.

Durante la búsqueda de antecedentes se encontraron investigaciones que estudian las transformaciones del discurso intercultural (Mateos Cortés, 2011); sin embargo, no se identificaron aportes que lo caractericen respecto de sus significaciones a nivel de planes de estudio en educación básica.

Dadas las anteriores condiciones, este ensayo ofrece una doble contribución al campo de conocimiento de la educación intercultural. Por un lado, cuestiona el lugar de este campo, el cual desatinadamente ha sido esencializado, folclorizado y reducido a temas de etnicidad. Lo anterior conduce entonces a sesgar la comprensión de la misma educación intercultural. Y, por el otro lado, se perfila como un modesto ejercicio historiográfico de la educación intercultural en la política educativa mexicana, lo que, a su vez, puede representar un aporte para las políticas interculturales de uso en América Latina, pues permite una reflexión profunda de la necesidad de derribar todo tipo de exclusión que se puede fomentar, incluso, en ambientes donde se promueve la educación intercultural.

\section{Producción documental del campo intercultural}

La educación intercultural en México se ha configurado con relación al corpus de proposiciones, sus pretensiones de verdad, el juego de reglas, las técnicas y los instrumentos (Foucault, 1973) que lo circundan. Es decir, existen condiciones históricas y sociales particulares ${ }^{3}$ que dieron paso a la emergencia del campo de la educación

\footnotetext{
${ }^{3}$ Algunos ejemplos de estos sucesos históricos son la Conquista, la Independencia de México, la Revolución mexicana, la creación de la SEP y la lucha del Ejercito Zapatista de Liberación Nacional (EZLN) para lograr el reconocimiento, el respeto y la educación de los sectores indígenas.
} 
intercultural. Siguiendo este argumento, es posible reconocer que la producción documental es tan diversa como el campo mismo.

A nivel nacional, los estados de conocimiento publicados por el Consejo Mexicano de Investigación Educativa (COMIE) son un referente obligado para la comprensión del campo. Referirse a dichos estados no tiene la pretensión de invizibilizar los enfoques internacionales (Dietz y Mateos Cortés, 2011) o los latinoamericanos (Comboni Salinas y Juárez Núñez, 2013) que igualmente nutren a la educación intercultural mexicana.

En el volumen tres "Educación, derechos sociales y equidad" (tomo I), correspondiente a la colección 1992-2002, se integró un apartado denominado "Educación y diversidad cultural". Los temas que allí se recuperan versan sobre "procesos de identidad cultural y social de los sujetos de intervención, experiencias pedagógicas, de formación y capacitación de agentes educativos, construcción de modelos educativos alternativos formales, la discusión filosófica, epistemológica y antropológica de las identidades sociales, y el multiculturalismo" (Medina Melgarejo, 2003, p. 147).

En la colección más reciente (2002-2011) el COMIE dedicó un volumen completo a las temáticas relacionadas con el multiculturalismo y la educación. De los productos allí sistematizados destacaron temas como lo transcontinental, la migración transnacional, la descolonización, los modelos de educación intercultural, la interculturalidad, la exclusión social y la educación comunal.

En esta edición se reconoció la existencia de dos tipos de investigaciones, una desarrollada de arriba hacia abajo - generadas en o desde las instituciones oficiales que regulan el currículo formal-, y la otra, de abajo hacia arriba -surgidas de proyectos locales y comunales- (Bertely Busquets, Dietz y Díaz Tepepa, 2013). Un rasgo característico de las investigaciones sistematizadas en ambos estados de conocimiento es que se inscriben mayormente en la educación básica o la superior (educación normalista y universidades interculturales); además, tienen un arraigo potente en temas de etnicidad, resistencia, migración, infancia, juventud y docencia.

Sin afán de presentar un ejercicio exhaustivo, lo anterior puede apreciarse con mayor detalle en los dos siguientes subapartados: a) huellas histórico-discursivas de lo intercultural, y b) construcciones escolarizadas del discurso intercultural. 


\subsection{Huellas histórico-discursivas de lo intercultural}

En este primer caso, destacan contribuciones que ubican a la educación intercultural en un marco de asimetría del poder en contextos globales y transcontinentales (Zemelman y Quintar, 2007), a través de posicionamientos apolíticos armónicos -en algunas agencias internacionales-, o conflictivos -en organizaciones indígenas- (González Apodaca, 2009).

Para ejemplificar lo anterior se señaló que las autoridades educativas de los modernos Estados-Nación "que se formaron después de la época colonial, emprendieron la tarea de establecer un sistema educativo formal, y pensaron resolver el problema del analfabetismo entre las poblaciones indígenas [...] mediante campañas de castellanización" (Amadio, 1987, p. 20). Para Amadio, este modelo integracionista representó una nueva forma de relación, en la que se incorporó a los sectores indígenas a la sociedad nacional. Algunos ejemplos de lo anterior fueron la Escuela Indígena de Warisata en Bolivia (1931) y el Proyecto Tarasco en México (1939), los cuales aceptaban por vez primera el ingreso de otras lenguas y manifestaciones culturales a la estructura educativa nacional. Respecto de estos ejemplos, se argumentó que "la educación y el nacionalismo van mano a mano, y en imitación a ideologías vigentes de esa época, se pensó que solo en base a una identidad uniforme, a una homogeneización cultural -por constituirse- podrían erigirse verdaderas sociedades nacionales" (Mayer, 1987, p. 32).

Dado lo anterior, se asumió que el desarrollo y la formación nacional de la sociedad centraba su atención en "un sector moderno capitalista (industria, comercio, agricultura de capital, sectores de servicios, etc.) y un sector obsoleto y atrasado conformado por campesinos indígenas y etnias pre-campesinas (tribales, según algunas terminologías)" (Varese, Gigante, Lewin y Pardo, 1987, pp. 39-40). Siguiendo el argumento anterior, este último sector tendría que alcanzar al sector moderno con la finalidad de obtener un beneficio propio, lo cual aseguraría un proceso de desarrollo e integración cultural de las etnias indígenas "a través de mecanismos directos (educación y capacitación) o indirectos (medios de comunicación, discriminación étnica, ejercicio jurídico y administrativo)" (Gigante, 1994, p. 40).

En otro orden de ideas, también se encontraron aportes que registraron el rechazo hacia las políticas nacionalistas que buscaban la asimilación de las poblaciones indígenas. Varese, Gigante, Lewin y Pardo (1987) ejemplificaron estos casos de resistencias a partir de la conformación de organizaciones indígenas independientes en Latinoamérica: el Modelo 
educativo MACAC en Ecuador, el Consejo Regional Indígena del Cauca (CRIC) en Colombia, la Alianza Nacional de Profesionales Indígenas Bilingües A.C. (ANPIBAC) en México y la Asociación Indígena de Costa Rica (ASINDIGENA).

Según el planteamiento anterior, Enrique Hamel (2000) afirma que, de la década de 1990 a la fecha, la relación entre pueblos indígenas y Estados-Nación, en América Latina, experimentó cambios considerables. Destaca los casos de adquisición de personalidad jurídica, territorios, derechos educativos y administrativos de las poblaciones indígenas brasileñas; la aprobación de una ley de educación indígena en Colombia; la promulgación de una ley de educación en Bolivia en 1994, la cual establecía a la interculturalidad para todo el país; la firma de acuerdos de Paz en Guatemala para incluir a los pueblos mayas, y el fortalecimiento del EZLN como un movimiento indígena insurreccional en México.

\subsection{Construcciones escolarizadas del discurso intercultural}

En este segundo caso, se recuperaron contribuciones que estudian la problematización de la educación intercultural en educación básica y su relación con el currículo (impuesto por el Estado o propuesto por ciertos sectores). El conjunto de aportes que aquí se presenta recupera estudios producidos "desde arriba" y "desde abajo".

Por un lado, respecto de los estudios "desde arriba", se encontró un trabajo que discute el desplazamiento de categorías. En el marco de la reforma educativa 2013, Mendoza Zuany señala que el concepto inclusión educativa suplió a la educación intercultural y bilingüe. En su análisis, reconoció que tal desplazamiento tiene "implicaciones visibles en rubros como la definición de beneficiarios, el presupuesto de programas, la oferta de educación inicial y continua de los docentes" (Mendoza Zuany, 2017, p. 52).

En este rubro se identificó un aporte que analiza las definiciones sobre la educación intercultural en los discursos normativos. Aguilar Nery privilegia el análisis de los documentos oficiales emitidos por el Gobierno mexicano y por el de Baja California. Concluye que estos discursos "siguen mostrando fallas y contradicciones, especialmente en lo relacionado con la definición de la cultura y en la distancia que guardan en la orientación de las prácticas escolares" (Aguilar Nery, 2004, p. 39).

Por el otro lado, están las contribuciones que recuperan experiencias curriculares "desde abajo". Aquí destacan estudios acerca de la resignificación del currículo nacional en contextos indígenas (Cano Ruiz, 2014); el rol de la escuela en las relaciones interétnicas 
(Leiva Guerrero, 2014); la relación entre libros de texto y diversidad cultural (Gigante, Díaz Couder, Pellicer y Olarte Tiburcio, 2001); las propuestas de escuelas denominadas interculturales, como es el caso del enfoque intercultural transversal en una telesecundaria suburbana del contexto veracruzano (Kreisel, 2016) o el de la telesecundaria Tetsijtsilin (Morales Espinoza, 2012); el trabajo en las escuelas multigrado rurales indígenas bajo un enfoque de doble inmersión (Feltes y Reese, 2014); las propuestas pedagógicas para incorporar saberes tradicionales al currículo nacional de ciencias naturales (Bascopé Julio y Caniguan Velarde, 2016); metodologías que permiten la co-teorización intercultural de un modelo de educación Intercultural y bilingüe en una escuela rural (Sartorello, 2014), así como reflexiones de la educación intercultural en el quehacer del profesional de la orientación educativa (Morales Trejo, 2015). Conviene decir que, por el interés de este ensayo, los estudios desarrollados "desde abajo" no son motivo de escrutinio detallado.

Si bien el campo de conocimiento de la educación intercultural es diverso, lo que es un hecho es que muchos de los productos citados previamente privilegian la condición indígena de la diversidad, aun tratándose del locus prescriptivo.

\section{Complexión escolarizada del discurso intercultural}

En México se reconocen tres enfoques de la Educación Intercultural, aunque hubo un giro nominal del segundo al tercero, su característica principal es que continúa (re) produciéndose con fuerte sesgo indigenista. En la Tabla 1 se presenta el periodo y la institución que cobijó cada uno de esos enfoques.

Tabla 1

Enfoques de la Educación Intercultural en México

\begin{tabular}{|c|c|c|c|}
\hline Periodo & $1963-1989$ & $1989-2009$ & $2001-2011$ \\
\hline Enfoque & $\begin{array}{c}\text { Educación Indígena } \\
\text { Bilingüe Bicultural (EIBB) }\end{array}$ & $\begin{array}{c}\text { Educación Intercultural y } \\
\text { Bilingüe (EIB) }\end{array}$ & $\begin{array}{c}\text { Educación Intercultural para } \\
\text { Todos (EIT) }\end{array}$ \\
\hline Institución & $\begin{array}{c}\text { Instituto Nacional } \\
\text { Indigenista (INI) }\end{array}$ & $\begin{array}{c}\text { Dirección General de } \\
\text { Educación Indígena } \\
\text { (DGEI) }\end{array}$ & $\begin{array}{c}\text { Coordinación General de } \\
\text { Educación Intercultural y } \\
\text { Bilingüe (CGEIB) }\end{array}$ \\
\hline
\end{tabular}

Fuente: Elaboración propia a partir de Diario Oficial de la Federación (DOF, 2001), Bello Domínguez (2009) y Martínez Buenabad (2011).

En la postrevolución, durante la segunda mitad del siglo XX, entre 1963 y 1964, el INI puso en marcha el Programa de Educación Indígena Bilingüe Bicultural, el cual marcó la institucionalización del enfoque EIBB. La política educativa que se impulsó fue la de "mexicanizar a los indios a través de la lengua nacional [...] la cual buscaba la asimilación de 
los indígenas por lo que sus culturas eran catalogadas como atrasadas" (Martínez Buenabad, 2011, p. 1) (sic).

Por su parte, con la creación de la DGEI en 1975, la SEP desarrolló una serie de métodos y contenidos educativos destinados a la población indígena, lo que incluyó la emisión del Programa de Educación Intercultural y Bilingüe. Este programa aseguró la puesta en marcha del enfoque de EIB y su propósito principal fue el de revitalizar las lenguas indígenas y sus espacios sociales de circulación (Bello Domínguez, 2009).

Finalmente, el enfoque de la EIT fue el resultado de la combinatoria entre historia nacional y recomendaciones de la Organización de las Naciones Unidas para la Educación, la Ciencia y la Cultura (UNESCO, por su significado en inglés) para generar políticas educativas. Entre ellas destacó la de promover y fortalecer "la educación intercultural y bilingüe en sociedades multiétnicas, plurilingües y multiculturales" (Unesco, 2000, p. 30). Para atender tal recomendación, primero se creó la CGEIB. Su principal atribución fue la de "promover y evaluar la política intercultural bilingüe en coordinación con las diferentes instancias del Sistema Educativo Nacional" (Diario Oficial de la Federación [DOF], 2001, p. 58). En segundo lugar, los gobiernos mexicanos de los tres últimos sexenios (2000-2006, 2006-2012 y 2012-2018) generaron una serie de políticas educativas que promovieron renovaciones curriculares en educación básica ${ }^{4}$, cuyo acento fue la educación intercultural (SEP, 2001, 2007, 2013). Para lograr lo anterior la CGEIB, la DGEI y la SEP trabajaron de manera colaborativa.

El breve repaso por los enfoques de la Educación Intercultural condujo a afirmar que a este tipo de educación la atraviesa la huella de lo indígena. Esta huella es "distinguida por Levinas del efecto, de la pista o del signo que no se relacionan con el otro como invisible absoluto" (Derrida, 1989, p. 138). Además, condujo a una cierta rehabilitación de la escritura como momento de convergencia más no de influencia; es decir, la huella retiene, guarda la

\footnotetext{
${ }^{4}$ Durante el primer sexenio se impulsó una "revisión y adecuación curricular de materiales educativos, así como de prácticas educativas en el aula y en la escuela, que dieran continuidad pedagógica y de contenidos a la educación básica, desde el nivel preescolar hasta la enseñanza secundaria" (SEP, 2001, p. 137) (sic). Lo anterior desembocó en el plan de estudios de Educación Preescolar 2004 y el de Educación Secundaria 2006. En el sexenio siguiente (2006-2012), y en razón del objetivo 1 del Programa Sectorial de Educación, se señaló la necesidad de "realizar una reforma integral de la educación básica, centrada en la adopción de un modelo educativo basado en competencias, que respondiera a las necesidades de desarrollo de México en el siglo XXI" (SEP, 2007, p. 23) (sic). Esto trajo como consecuencia dos renovaciones curriculares, el Plan de estudios de Educación Primaria 2009 y la RIEB 2011. Finalmente, durante el periodo sexenal que recién finalizó en diciembre del año pasado (2012-2018), el objetivo 1 del Programa Sectorial de Educación fue "garantizar la pertinencia de los planes y programas de estudio, así como los materiales educativos” (SEP, 2013, p. 45). Esto resultó en la publicación del MEpEO 2017, el cual se encuentra vigente desde agosto de 2018.
} 
palabra, la obra; indica una proximidad con el otro, los mundos otros. Dicho de manera distinta, es lo que permite revisar la elaboración de la educación intercultural, de sus condiciones de posibilidad y su prospectiva. Como se verá en los siguientes subapartados, la huella indígena es la que predomina en la configuración del discurso intercultural.

En la tabla 2 se presenta la complexión escolarizada del discurso intercultural, ésta fue el resultado del análisis hecho a los planes de estudio renovados en lo que va de este siglo.

Tabla 2

Complexión escolarizada del discurso intercultural en planes renovados de básica

\begin{tabular}{|c|c|c|c|c|}
\hline \multirow[b]{2}{*}{$\begin{array}{l}\text { Plan de } \\
\text { estudios }\end{array}$} & \multicolumn{3}{|c|}{ Tipos de significantes } & \multirow{2}{*}{$\begin{array}{l}\text { Articulador } \\
\text { común }\end{array}$} \\
\hline & $\begin{array}{l}\text { Asociados con la } \\
\text { Educación }\end{array}$ & $\begin{array}{l}\text { Con sufijos } \\
\text { "idad/ismo" }\end{array}$ & Adjetivantes & \\
\hline $\begin{array}{l}\text { Preescolar } \\
2004\end{array}$ & $\begin{array}{l}\text { Educación Bilingüe e } \\
\text { Intercultural }\end{array}$ & $\begin{array}{c}\text { Diálogo } \\
\text { intercultural }\end{array}$ & - & \multirow{5}{*}{$\begin{array}{l}\text { Diversidad } \\
\text { social, } \\
\text { étnica, } \\
\text { cultural y } \\
\text { lingüística }\end{array}$} \\
\hline $\begin{array}{l}\text { Secundaria } \\
2006\end{array}$ & $\begin{array}{c}\text { Educación Intercultural } \\
\text { para Todos }\end{array}$ & Interculturalidad & $\begin{array}{l}\text { Conciencia } \\
\text { Intercultural }\end{array}$ & \\
\hline $\begin{array}{l}\text { Primaria } \\
2009\end{array}$ & $\begin{array}{c}\text { Atención Educativa } \\
\text { Intercultural y Bilingüe }\end{array}$ & Interculturalidad & $\begin{array}{c}\text { Realidad } \\
\text { Intercultural }\end{array}$ & \\
\hline $\begin{array}{l}\text { Plan de } \\
\text { educación } \\
\text { básica } 2011\end{array}$ & $\begin{array}{c}\text { Educación Intercultural y } \\
\text { Bilingüe }\end{array}$ & Interculturalidad & $\begin{array}{c}\text { Nación } \\
\text { Multicultural } \\
\text { Relaciones } \\
\text { Interculturales } \\
\text { Contexto } \\
\text { Intercultural } \\
\text { Bilingüe o } \\
\text { Trilingüe } \\
\text { Modelo } \\
\text { Intercultural }\end{array}$ & \\
\hline $\begin{array}{l}\text { Modelo } \\
\text { Educativo } \\
2017\end{array}$ & $\begin{array}{c}\text { Educación Intercultural y } \\
\text { Bilingüe }\end{array}$ & $\begin{array}{l}\text { Interculturalidad } \\
\text { Multiculturalidad }\end{array}$ & $\begin{array}{c}\text { Grupos } \\
\text { Multiculturales }\end{array}$ & \\
\hline
\end{tabular}

Fuente: Elaboración propia a partir de SEP $(2004,2006,2009,2011,2017)$.

Con ayuda de la tabla 2 se identificaron cuatro hallazgos. Primero, cada plan de estudios aglomera distintos significantes, los cuales pueden agruparse en tres categorías: a) asociados con la educación, b) con sufijos "idad/ismo" y c) adjetivantes. Segundo, independientemente de que estos significantes presenten similitudes nominales, se antagonizan y contraponen con las significaciones que caracterizan a cada plan y, por tanto, se reconoce la existencia de una relación constitutiva entre significaciones. Tercero, lo anterior comunica una estabilidad temporal de significantes, significaciones y sectores de 
atención que han sido el resultado de contingencias histórico-contextuales. De ahí que convenga afirmar que esta complexión no es última, sino que se nutre dependiendo de la entrada, la salida o la combinación de significantes, significaciones y sectores de atención. Cuarto, en todos los planes de estudio la noción diversidad (social, étnica, cultural, lingüística, etc.) fue recurrente, juega el papel de articulador común, aunque casi siempre se reduce a temas de etnicidad.

En los subapartados siguientes se desagrega el análisis de la complexión escolarizada del discurso intercultural. De manera sistemática, se analiza cada plan de estudios. En cada caso se ofrecen aspectos organizativos de dichos planes, posteriormente se destacan las citas textuales donde se ubicaron los significantes apuntados en la Tabla 2, y finalmente, se presenta una discusión de sus significaciones y los sectores de atención que cada uno postula.

\subsection{Preescolar 2004. Educación para el diálogo intercultural}

En este plan de estudios ${ }^{5}$ se asume que los primeros años de vida de una persona constituyen un periodo "intenso de aprendizaje y desarrollo que tiene como base la propia constitución biológica o genética, pero en el cual desempeñan un papel clave las experiencias sociales, es decir, la interacción con otras personas, ya sean adultos o niños" (SEP, 2004, p. 12). En la Tabla 2 se indicaron dos tipos de significantes del discurso intercultural para preescolar, uno asociado con la noción educación (educación bilingüe e intercultural) y otro de tipo adjetivante (diálogo intercultural).

El primer significante -educación bilingüe e intercultural- se encontró en el apartado "La educación: un derecho fundamental", de dicho plan. En él se argumentó que la educación se consideraba un servicio público de interés social, en ese contexto se señaló "como obligaciones de las autoridades, entre otras, la de favorecer la educación bilingüe e intercultural e impulsar el respeto y conocimiento de las diversas culturas existentes en la nación" (SEP, 2004, p. 16). Según este plan de estudios, las culturas eran concebidas por la SEP como aquellas que podían "estar asociadas con la pertenencia a un grupo étnico [...] En

\footnotetext{
${ }^{5}$ Se promulgó durante el primer periodo gubernamental de este siglo (2000-2006). El documento se compone por siete apartados: a) fundamentos: una educación preescolar de calidad para todos; b) características del programa; c) propósitos fundamentales; d) principios pedagógicos; e) campos formativos y competencias; f) organización del trabajo docente durante el año escolar; y g) evaluación.
} 
los grupos étnicos, una característica central es una lengua materna propia, con grados distintos de preservación y de coexistencia con el español" (SEP, 2004, p. 37).

Lo dicho en la cita anterior resultó revelador porque la SEP sostiene la conceptualización de una cultura dogmática e incontaminable, en la que no alcanzan a reconocerse los entre-medios (Bhabha, 1996), es decir, las resistencias y las negaciones dentro de esa misma conceptualización.

Al respecto conviene hacer dos preguntas ¿acaso no hay cultura afuera de lo étnico? y ¿qué es lo que les da identidad (Hall, 1996) a esas personas que se asumen como parte de determinados sectores de la población (sean o no indígenas)? Es notorio que a lo largo del plan 2004 se invisibilizaron las (otras) diversidades que se articulan a la educación intercultural.

En cuanto al sector de atención, es de considerarse que, si se trata de una educación bilingüe e intercultural, los contenidos curriculares desagregados en dicho plan no contemplaron el aprendizaje de algún idioma (denominado) indígena, la lengua de señas o el Braille. Por lo contrario, la SEP reitera la condición folclorizada y reduccionista de las culturas (creencias, tradiciones, costumbres, grupo específico, etc.).

Del segundo significante -diálogo intercultural- se ubicaron dos significaciones. Una, en el apartado "El programa tiene un carácter nacional", en el que se señaló que dicho diálogo se promovía en los salones de clase; y la otra significación en el apartado "Principios pedagógicos", específicamente, en el subtema "Diversidad y equidad". Aquí, el diálogo intercultural se esbozó como un elemento cuyo desarrollo dependía de la acción docente respecto de las condiciones geopolíticas en las que se encontrara el alumnado, sus estilos de aprendizaje, etc. (SEP, 2004).

En este plan de estudios el alumnado de preescolar se concibió como el sector de atención de la Educación Intercultural. De acuerdo con este análisis, la SEP reconoció en la escolarización la posibilidad del reconocimiento de las culturas del país y el respeto a la diversidad. 


\subsection{Secundaria 2006. La trama de la interculturalidad}

En este plan $2006^{6}$ se indicó que, "ya sea que continúen con una educación formal o ingresen al mundo laboral, la escuela secundaria asegurará a los adolescentes la adquisición de herramientas para aprender a lo largo de toda su vida" (SEP, 2006, p. 8). En este sentido, a la Educación Secundaria se le asignó la tarea de contribuir con la formación ciudadana bajo una perspectiva democrática y productiva en la vida del país. En el contenido de este plan se hallaron tres tipos de significantes asociados con la Educación Intercultural: uno ligado a la educación (educación intercultural para todos), otro con sufijo "idad/ismo" (interculturalidad) y uno adjetivante (conciencia intercultural).

Respecto del primer significante -educación intercultural para todos-, en el apartado "Las finalidades de la educación básica", se explicó que México es un país multicultural que "asume la existencia de diferentes culturas, etnias y lenguas, y requiere, por tanto, impulsar una educación intercultural para todos, que identifique y valore esta diversidad y, al mismo tiempo, afirme su identidad nacional" (SEP, 2006, p. 7). Según lo anterior, esta diversidad no se perfiló como exclusiva de un sector poblacional, ni mucho menos se restringió a las características somáticas de las personas. Sin embargo, es impensable que la configuración de México se separe de sus condiciones históricas y de la huella indígena. Lo anterior se encuentra sustentado en el artículo 2 de la Constitución política mexicana.

La Nación tiene una composición pluricultural sustentada originalmente en sus pueblos indígenas que son aquellos que descienden de poblaciones que habitaban en el territorio actual del país al iniciarse la colonización y que conservan sus propias instituciones sociales, económicas, culturales y políticas, o parte de ellas [...] de acuerdo con sus usos y costumbres. (DOF, 2016, p. 2)

El segundo significante -interculturalidad- se ubicó en el apartado "Características del plan y de los programas de estudio". La interculturalidad fue anunciada como una propuesta "para mejorar la comunicación y la convivencia entre comunidades con distintas culturas, siempre partiendo del respeto mutuo. Esta concepción desde las asignaturas, se tradujo en

\footnotetext{
${ }^{6} \mathrm{Al}$ igual que el de preescolar, el plan de secundaria se aprobó durante el primer periodo presidencial de este siglo. Los siete apartados que lo integran son: a) las finalidades de la educación básica; b) perfil de egreso de la educación básica; c) elementos centrales en la definición de un nuevo currículo; d) características del plan y de los programas de estudio; e) mapa curricular; f) propósitos de las asignaturas; g) orientaciones didácticas para el mejor aprovechamiento de los programas de estudio.
} 
propuestas prácticas de trabajo en el aula, sugerencias de temas y enfoques metodológicos" (SEP, 2006, p. 19) (sic). En este entramado destacó la formación en valores, la educación sexual y la equidad de género.

Concretamente, la propuesta curricular 2006 pretendía desarrollar competencias relacionadas con la mejora de las relaciones interpersonales entre dos sectores, los cuales no fueron mencionados explícitamente; sin embargo, por intertextualidad (Kristeva, 1978) se identificó que se trataba de la población indígena y la no indígena. Al respecto, resulta importante destacar que la interculturalidad es factible de presentarse al interior de las regiones indígenas y las no indígenas, en función de las diversidades existentes en cada una de esas regiones. En la Tabla 3 se ejemplifica lo anterior.

Tabla 3

Contenidos curriculares para favorecer la Educación Intercultural en secundaria

\begin{tabular}{|c|l|}
\hline Asignatura & \multicolumn{1}{c|}{ Contenidos curriculares } \\
\hline Español & $\begin{array}{l}\text { Riqueza lingüística y cultural de México } \\
\text { Variedades sociolingüísticas del español }\end{array}$ \\
\hline Ciencias & $\begin{array}{l}\text { Manifestaciones culturales } \\
\text { Multiculturalidad como parte de la formación de la identidad nacional }\end{array}$ \\
\hline Historia & $\begin{array}{l}\text { Aportes de los pueblos al patrimonio cultural } \\
\text { Origen de los rasgos de México como nación multicultural }\end{array}$ \\
\hline $\begin{array}{c}\text { Formación } \\
\text { cívica y ética }\end{array}$ & $\begin{array}{l}\text { Imagen positiva de sí mismos } \\
\text { Pluralidad y respeto a la diversidad como principios para una convivencia } \\
\text { democrática } \\
\text { Resolución de conflictos de manera pacífica }\end{array}$ \\
\hline $\begin{array}{c}\text { Artes. } \\
\text { Música }\end{array}$ & Respeto a la diversidad musical (México y otros países) \\
\hline $\begin{array}{c}\text { Artes. Artes } \\
\text { visuales }\end{array}$ & $\begin{array}{l}\text { Solidaridad en el trabajo colectivo } \\
\text { Respeto a la diversidad cultural }\end{array}$ \\
\hline
\end{tabular}

Fuente: Elaboración propia a partir de SEP (2006).

Los contenidos de la Tabla 3 albergan dos cadenas de equivalencia. Se entenderá por cadena de equivalencia a aquella que "sólo es válida para determinadas posiciones en el interior de un contexto estructural dado [...] por un lado conserva su propio sentido literal; por el otro simboliza la posición contextual respecto a la cual es un elemento sustituible" (Laclau y Mouffe, 1987, pp. 109-110). En estricto sentido, cada cadena se conforma por nociones que están asociadas con un significante. Según se señala en la Tabla 3, la primera cadena que se identificó es "regiones indígenas". Las nociones que a ella se asocian son: riqueza lingüística y cultural, pueblos, variedades sociolingüísticas, origen de los rasgos de México, imagen positiva de sí mismos y manifestaciones culturales. La segunda cadena de 
equivalencia que se ubicó es "población no indígena". Las nociones que a ella están ligadas son: resolución pacífica de conflictos, solidaridad y convivencia democrática.

Como se señaló previamente, la SEP no alude a las diversidades al interior de las regiones consideradas o no indígenas. No obstante, este análisis indica que los intersticios dialógicos de esta complexión están constituidos por tres elementos: la resolución pacífica de conflictos, la solidaridad en el trabajo colectivo y la convivencia democrática, los cuales confirman la existencia de una producción de sentido diversificante de lo indígena (interior) desde lo no indígena (exterior).

El tercer significante -conciencia intercultural- se encontró en el apartado "Propósitos de las asignaturas", específicamente, en Ciencias I (Biología). La conciencia intercultural se planteó como la posibilidad de comprender que el conocimiento científico es producto de la reflexión y de las aportaciones de personas de distintas culturas (SEP, 2006). El acento se colocó en los presupuestos del discurso de equidad de género, donde se reconoce a mujeres y hombres como catalizadores del saber.

A pesar de lo anterior, el asunto problemático identificado es que en el plan 2006 poco se alude a otros tipos de saberes o cosmovisiones (regionales, locales, comunitarios, etc.), en los que la ciencia ha ido encontrado respuestas a cuestiones de salud, alimentación, de afectaciones meteorológicas, etc. Sin duda, lo anterior da cuenta de una cierta incapacidad de la SEP respecto del diseño de la política educativa, pues las pedagogizaciones que realiza de la diversidad tienden a cosificar a las regiones indígenas. Siguiendo este argumento, lo anterior conlleva a un tipo de racismo sofisticado en el que se excluye al incluido, y donde se multiplican las posibilidades de discriminación más que a las de respeto y de reconocimiento.

\subsection{Primaria 2009. Hacia la comprensión de una realidad intercultural}

En este plan ${ }^{7}$ se indicó que, en relación con los compromisos internacionales, en México, la escolaridad obligatoria abarca "la educación primaria y secundaria e incluso algunos sistemas educativos, como el caso mexicano, han incorporado uno, dos o más años

\footnotetext{
7 Se aprobó durante el segundo periodo sexenal de este siglo (2006-2012). Su estructura contempla siete apartados: a) la educación básica en el contexto internacional y nacional; b) principales retos para ofrecer educación de calidad; c) elementos centrales en la definición del nuevo currículo; d) la articulación curricular de la educación básica; e) competencias para la vida y perfil de egreso de la educación básica; f) características del plan y los programas de estudio; y g) mapa curricular.
} 
como obligatorios en la educación preescolar, con el propósito de alcanzar un mejor logro en las siguientes etapas" (SEP, 2009, p. 10).

Siguiendo el contenido de la Tabla 2, el discurso intercultural en el plan de estudios 2009 está integrado por tres significantes: uno asociado con la educación (atención educativa intercultural y bilingüe), otro con sufijo "idad/ismo" (interculturalidad) y, el último, adjetivante (realidad intercultural). El primer significante -atención educativa intercultural y bilingüe- se halló en el apartado "Principales retos para ofrecer educación de calidad". En él se explicó que "no sólo se requieren estrategias curriculares y de gestión escolar pertinentes, también hacen falta maestros que hablen las lenguas maternas del estudiantado, así como materiales de apoyo que contribuyan a la mejora educativa de estos grupos poblacionales" (SEP, 2009, p. 21), es decir, los denominados indígenas. Conforme a estos planteamientos, la atención educativa intercultural y bilingüe puso de manifiesto una secularización de la alfabetización a través de la castellanización. Este tipo de atención enfatizó la importancia de la relación dialógica entre hablantes indígenas y no indígenas.

Avanzando en la lectura de este plan 2009, se encontró que la atención educativa intercultural y bilingüe estaba dirigida a hijas e hijos de jornaleros agrícolas migrantes. Al respecto, la Secretaría de Educación indicó que "desde hace 26 años, esto es, desde 1982, la Secretaría de Educación Pública viene promoviendo la atención educativa intercultural en primaria y preescolar" (SEP, 2009, p. 22). Estos otros sectores socioeconómicos (migrantes, jornaleros agrícolas migrantes, etc.) viraron la atención de lo intercultural y bilingüe. Su visibilización evidenció la ampliación del proceso de inclusión, el cual fue el resultado de múltiples demandas sociales. Actualmente, estas demandas han alcanzado a las regiones afrodescendientes. No obstante, su inclusión político-normativa está ausente en la Constitución política mexicana.

En el apartado "Características del plan y los programas de estudio" se identificó al segundo significante -interculturalidad-. Este se definió como un tipo de "riqueza y forma de convivencia en la diversidad social, étnica, cultural y lingüística [...] así como tomar en cuenta las distintas expresiones de la diversidad que caracterizan a nuestro país y a otras regiones del mundo" (SEP, 2009, pp. 43-44). En este plan 2009, al igual que el de secundaria 2006, la interculturalidad se concibió como una propuesta de comunicación y convivencia entre personas. En este caso, la SEP esperaba que el estudiantado de 
Educación Primaria, en su condición de sujetos sociales, reconociera y valorara la diversidad social y cultural que caracteriza a México.

Finalmente, en cuanto al tercer significante -realidad intercultural-, el plan de estudios en cuestión consideró que una realidad intercultural tenía como eje

[...] el establecimiento de una relación diferente entre grupos humanos con culturas distantes, donde la primera condición es la presencia de una relación de igualdad, modulada por el principio de equidad; la segunda es reconocer al otro como diferente, sin menospreciarlo, juzgarlo o discriminarlo, sino sobre todo comprenderlo y respetarlo; la tercera es ver a la diversidad cultural como riqueza. (SEP, 2009, p. 175)

Es posible comprender que el alumnado, y su pertenencia a distintos grupos culturales, se consideraró como el sector de atención. Según el plan 2009, la inclusión y el reconocimiento solo son posibles a través de la acción docente en las aulas de primaria, de tal manera que se buscó que el alumnado apreciara esta diversidad. Tal como se señala en la cita, este aprecio de la diversidad se bosqueja en el marco de una "relación diferente", es decir, simétrica y horizontal, que apunte al desvanecimiento de las jerarquías y los estereotipos mediante el diálogo.

En este ensayo se concibe al diálogo como una desventaja para aprender, es decir, como contingencia. La contingencia "se refiere al evento imprevisto o característica extraordinaria que modifica el carácter del objeto. Su estatus es constitutivo, no de desviación ni parasítico de una esencia permanente" (Buenfil Burgos, 2009, p. 102). El diálogo no tiene pretensión de ser armónico, pero casi siempre conlleva una suerte de desventaja respecto de quienes dialogan. Al ser una contingencia, el diálogo representa un área de oportunidad para aprender del otro y para situarse como sujeto social, pero, sobre todo, para reivindicar la propia condición humana desde las diferencias que nos constituyen como humanidad.

\subsection{Plan de educación básica 2011 ¿Hacia un modelo intercultural?}

En el plan $2011^{8}$, la SEP señaló que "la RIEB culmina un ciclo de reformas curriculares en cada uno de los tres niveles que integran la Educación Básica [...] y consolida este

\footnotetext{
${ }^{8}$ Al igual que el de primaria, la Reforma Integral de la Educación Básica (RIEB) también se aprobó durante el segundo periodo sexenal. Esta reforma trajo consigo la publicación del plan de estudios de Educación Básica 2011, el cual está organizado en dos secciones. La primera, "Reforma Integral de la Educación Básica", y la
} 
proceso, aportando una propuesta formativa pertinente, significativa, congruente, orientada al desarrollo de competencias" (SEP, 2011, p. 8 y 9). Como se señala en la Tabla 2, la complexión escolarizada se constituyó por seis significantes. Uno asociado con la educación (Educación intercultural y bilingüe), uno con sufijo "idad/ismo" (Interculturalidad) y cuatro adjetivantes (Nación multicultural, Relaciones interculturales, Contexto intercultural bilingüe o trilingüe y Modelo intercultural).

En cuanto al primer significante -educación intercultural y bilingüe- se señaló que "la lengua indígena se integra con la enseñanza del Español como segunda lengua, cumpliendo el mandato constitucional de ofrecer una educación intercultural y bilingüe, y de avanzar hacia la construcción de una nación plural" (SEP, 2011, p. 47). Siguiendo esta lectura, se consideró que dicho significante puso de manifiesto el conocimiento de cualquier otra cultura (intercultural), a través de la dimensión lingüística (bilingüe). Conviene destacar que, en este plan, la lengua indígena se colocó en igualdad de condiciones respecto del idioma español, esto le dio cierta visibilidad, reconocimiento y protagonismo respecto de otros momentos históricos nacionales. No obstante, hasta la fecha, los idiomas (denominados) indígenas no se han integrado al currículo nacional para su enseñanza, más bien, se reajustan en las distintas regiones indígenas (rediseños, traducciones, cuadernos de trabajo, etc.). Aunque es tema de otro debate, convendría saber qué están haciendo las normales públicas del país o las distintas unidades de la Universidad Pedagógica Nacional para formar docentes que dominen alguno de los idiomas indígenas.

El segundo significante -interculturalidad-, se ubicó en el subapartado "Diversificación y contextualización curricular". En el plan 2011, se señaló que los Marcos curriculares

[...] permiten acceder a la indagación, profundización e inclusión de los conocimientos de los pueblos y las comunidades desde la perspectiva derivada de su cosmovisión [...] Los saberes locales se plantearán desde un enfoque valorativo de lo propio que ayude a desarrollar una identidad positiva en la niñez indígena, que le permita acceder a la interculturalidad con bases culturales bien cimentadas y establecer relaciones

segunda, "Características del Plan de estudios 2011". Esta última se constituyó por nueve subapartados: a) principios pedagógicas que sustentan el plan de estudios; b) competencias para la vida; c) perfil de egreso de la Educación Básica; d) mapa curricular de la Educación Básica; e) diversificación y contextualización curricular: marcos curriculares para la educación indígena; f) parámetros curriculares para la educación indígena; g) gestión para el desarrollo de Habilidades Digitales; h) la gestión educativa y de los aprendizajes; i) estándares curriculares y aprendizajes esperados. 
simétricas con grupos 0 individuos de otras culturas, enriquecerse con los conocimientos de otros, dialogar y aportar sus saberes. (SEP, 2011, p. 58)

Dadas estas condiciones, la interculturalidad se conceptualizó como un lugar de llegada, es decir, un estadio ideal, algo para lo cual se trabaja. Las condiciones de producción para el logro de esta interculturalidad se sitúan en una cadena de equivalencia productora (conocimientos, saberes locales, cosmovisión, lo propio e identidad positiva). Lo anterior pone de manifiesto la equidad (relaciones simétricas) como aspecto aspiracional en la relación indígenas-no indígenas a través del diálogo, la interacción y el reconocimiento. En este sentido, el enriquecimiento de saberes representa, para la SEP, una condición constitutiva de la interculturalidad, y cuyo desarrollo se configura en la escuela y en las aulas.

Por su parte, en la introducción del plan de Educación Básica 2011 se indicó que el tercer significante -nación multicultural- buscaba el favorecimiento de "la conciencia de vivir en un entorno internacional insoslayable [...] También precisa fomentar en los alumnos el amor a la Patria y su compromiso de consolidar a México como una nación multicultural, plurilingüe, democrática, solidaria y próspera" (SEP, 2011, p. 12). La lectura realizada indica que la educación permitiría que el estudiantado se reconociera como parte de una nación multicultural, en la que se forman en la convivencia para identificarse como integrantes de una ciudadanía global en contextos locales. No obstante, la SEP obvió que el horizonte de formación nacional no es equitativo.

El cuarto significante -relaciones interculturales- se identificó en el subapartado "Favorecer la inclusión para atender a la diversidad". El plan 2011 señala que la educación ofrecía oportunidades para instrumentar "las relaciones interculturales, reducir las desigualdades entre grupos sociales, cerrar brechas e impulsar la equidad" (SEP, 2011, p. 35). En tal caso, dichas relaciones se concibieron como una posibilidad de la diversidad para trabajar la equidad. Como lo señala este plan, la educación mexicana ofrece los mecanismos necesarios para el diálogo entre diferentes, las oportunidades dentro de las desigualdades y la vigencia de la diversidad misma. Se encontró que, para el logro efectivo de estas relaciones interculturales, la SEP ofreció la educación inclusiva como un postulado para la comunicación de las diferencias. Lo anterior tiene una relación estrecha con lo que Mendoza Zuany (2017) indicó acerca del desplazamiento de los discursos. 
El quinto significante -contexto intercultural bilingüe o trilingüe- se halló en el apartado "Mapa curricular de la Educación Básica". En cuanto a los campos de formación de la Educación Básica, se expresó la necesidad de que

[...] los responsables de las asignaturas vinculadas a la enseñanza del lenguaje (Español, Lengua indígena e Inglés) hicieran del aula un espacio de encuentro entre las lenguas y sus culturas; es decir, que propicien un contexto intercultural bilingüe o trilingüe (en el caso de las escuelas indígenas) que se aproveche y explote para los aprendizajes lingüísticos y culturales de los estudiantes. (SEP, 2011, p. 45) (sic)

En relación con el argumento que se sostiene en este ensayo, la SEP buscó democratizar el encuentro de las culturas a través de la condición lingüística de los seres humanos. Colocó a los idiomas indígenas en un plano de igualdad frente al español y el inglés. Sin embargo, su inclusión solo alcanzó el estatus de asignatura para las escuelas primarias ubicadas en regiones indígenas.

Finalmente, el sexto significante -modelo intercultural- se encontró en la siguiente cita:

[...] la asignatura de Lengua indígena forma parte de un modelo intercultural, se consideran prácticas relacionadas con la diversidad cultural y lingüística que permiten a los niños comprender que su lengua es una entre otras tantas que hay en el país, y tiene el mismo valor que el español y las demás lenguas indígenas. (SEP, 2011, p. 46)

En la narrativa de la SEP, aludir al modelo intercultural es centrarse en el alumnado indígena para el reconocimiento de sí, desde sí. La enseñanza de los idiomas indígenas solo para indígenas pone en desventaja a este tipo de poblaciones, toda vez que se trata de una forma de aporía (Derrida, 1998) en la que se incluye de manera enunciativo-nominal y, a su vez, se les excluye del proceso generalizado de educación nacional. Esta aporía da cuenta de un proceso de desidentificación indígena y de indignidad nacional debido a razones somáticas.

\subsection{MEpEO 2017 ¿Educación Intercultural en el Nuevo Modelo de Básica?}


La SEP asume que el Modelo Educativo $2017^{9}$ "tiene como fin último colocar una educación de calidad con equidad donde se pongan los aprendizajes y la formación de niñas, niños y jóvenes en el centro de todos los esfuerzos educativos" (SEP, 2017, p. 27). En materia de Educación Intercultural, como se señala en la Tabla 2, el MEpEO 2017 albergó cuatro significantes, uno asociado con la educación (Educación intercultural y bilingüe), dos con sufijo "idad/ismo" (Interculturalidad y Multiculturalidad) y uno adjetivante (Grupos multiculturales).

Respecto del primer significante -educación intercultural y bilingüe-, en el apartado "inclusión y equidad", se señaló que los principales nudos de desigualdad se encuentran en la educación destinada a indígenas, jornaleros agrícolas migrantes y en los servicios que ofrece el Consejo Nacional de Fomento Educativo (CONAFE). En consecuencia, la SEP consideró que, para atender este desafío, era necesario un esfuerzo de focalización que considerara "realizar una planeación lingüística, que va desde reconfigurar la oferta inicial de educación intercultural y bilingüe, para asegurar que los docentes tengan un mejor dominio de la lengua en que enseñen, hasta las necesidades escolares actuales" (SEP, 2017, pp. 158-159).

Distinto de los cuatro planes de estudio anteriores, el magisterio se perfiló como el sector de atención en el MEpEO 2017. Si bien la SEP concibió a las poblaciones migrantes e indígenas como vulnerables y susceptibles de discriminación, no podrían atenderse en la ausencia de una formación lingüística (bilingüe) por parte de quienes se dedican a la docencia.

El segundo significante -interculturalidad- se encontró en la introducción del MEpEO 2017; específicamente, en lo que respecta al perfil de egreso del estudiante. Aquí la SEP destacó la necesidad de "reconocer que la diversidad tiene lugar en un espacio democrático, con inclusión e igualdad de derechos de todas las personas. Entiende las relaciones entre sucesos locales, nacionales e internacionales, valora y practica la interculturalidad" (SEP, 2017, p. 50). En este sentido, la SEP concibió a la interculturalidad como un fin pragmático y sustentable, toda vez que en cada nivel educativo el alumnado tendría que reconocer sus

\footnotetext{
${ }^{9}$ Se publicó durante el periodo sexenal que recientemente finalizó (2012-2018) y en el marco de la cuestionada Reforma Educativa 2013. Si bien, esta reforma no es motivo de discusión, en este ensayo se asume que se trata de un mecanismo de estímulo respuesta, es decir, de sumisión y sujeción de las personas docentes de Educación Básica y Media Superior. Regresando al tema que nos compete, el MEpEO 2017 está integrado por cinco apartados: a) el planteamiento curricular; b) la escuela al centro del sistema educativo; c) formación y desarrollo profesional de los maestros; d) inclusión y equidad; e) la gobernanza del sistema educativo.
} 
condiciones familiares, su identidad personal y su identidad nacional para alcanzar dicha interculturalidad y, en consecuencia, reducir el racismo y la discriminación.

El tercer significante -multiculturalidad- se reconoció como una extensión de lo argumentado previamente. La SEP asumió que la planeación lingüística de quienes se forman para ser docentes tendría que partir del "bilingüismo, el plurilingüismo, la multiculturalidad y las aulas multigrado, para permitir la atención de necesidades específicas de aprendizaje en las lenguas de dominio de los estudiantes, ya sean indígenas, español, Lengua de Señas Mexicana, o una lengua extranjera" (SEP, 2017, p. 159). Por intertextualidad puede asumirse a la multiculturalidad como un dispositivo más amplio, con el que se pueden visibilizar las otras posibilidades de la diversidad (idiomas distintos al indígena y el español). Por tanto, el diálogo en la diversidad se resignificó respecto de la condición lingüística de los seres humanos. Aunque como ya se señaló con anterioridad, el diálogo es contingente.

Finalmente, al cuarto significante -grupos multiculturales- se le encontró en el apartado "Inclusión y equidad". Respecto de este significante, la SEP expuso que entre sus principales objetivos estaban la posibilidad de que el estudiantado aprendiera "a convivir, trabajar y relacionarse en grupos multiculturales, bilingües, plurilingües, de contextos económicos y lugares de origen distintos [...] y comprendan la diversidad como una fuente de enorme riqueza" (SEP, 2017, p. 154). Por consiguiente, los grupos multiculturales resultan de, cuando menos, tres situaciones: a) la imbricación sociocultural; b) los esquemas de pensamiento; y c) las relaciones simbólicas e interpersonales.

En este análisis la diversidad se perfiló como condición constitutiva, es decir, como la "multiplicidad de formas de pensamiento, sentimiento y cultura. Puede presentarse dentro de un salón de clases en el que la totalidad del alumnado pertenezca a una misma cultura, pero cada quien presenta capacidades cognitivas, emocionales o sociales distintas" (SEP, 2017, p. 204). Bajo esta lógica, la Educación Intercultural puede asumirse como un dispositivo de anudamiento, relación, diálogo y comunicación entre pares, sin importar su procedencia cultural, social o política.

\section{Discusión de la complexión escolarizada del discurso intercultural}

El análisis presentado en este ensayo permitió identificar cuatro hallazgos. Primero, con la publicación y el desarrollo de los planes de estudio, las escuelas de educación básica 
se convirtieron (prescriptiva o enunciativamente) en lugares de la actividad intercultural. En consecuencia, las prácticas educativas de quienes se dedican a la docencia le asignaron una carga social destacada al alumnado. A través de éste y de su cotidianidad como ciudadanos, podría corroborarse la apuesta de la SEP por la implementación de la Educación Intercultural. En tal caso, la escuela se invistió como un lugar común de (des) encuentros y de inscripción para la alfabetización intercultural.

Segundo, el análisis de la complexión escolarizada del discurso intercultural permite observar la existencia de puntos de fuga que, en sentido Deleuziano, imposibilitan los cerramientos así como la irreductibilidad de las significaciones y del conocimiento. A partir de la complexión escolarizada (la que corresponde a cada plan de estudio y la que da cuenta del conjunto de los planes renovados) es posible percatarse de que los planes de estudio fungen como dispositivos de sujeción docente, donde "el poder regulador no sólo actúa sobre un sujeto preexistente, sino que también labra y forma al sujeto [...] es también estar subjetivado por él, es decir, devenir como sujeto precisamente a través de la reglamentación" (Butler, 2006, p. 68). De ahí que, como se expresó al inicio de este ensayo, la docencia se encuentra sujetada a los ordenamientos regulatorios de la SEP. Las personas que se dedican a esta profesión tienen la encomienda de diseminar un cierto discurso intercultural alineado a una agenda nacional o internacional, según sea el caso. Aunque esto no limita su libertad de cátedra.

Tercero, en los planes de estudio analizados, los sectores de atención de la Educación Intercultural escolarizada se subjetivan en las aulas de clase. De acuerdo con lo anterior, el alumnado se concibe como catalizador de ciudadanía, justicia social y tolerancia frente a la diversidad. No obstante, es evidente que prevalece el sesgo indígena en los documentos analizados, lo cual responde en buena medida a la configuración histórica de México.

Este discurso intercultural prescriptivo proyecta una cierta otredad en sus distintas líneas operativas (el perfil de egreso de educación básica, los propósitos del nivel educativo, los contenidos y enfoques curriculares, por citar algunos ejemplos). Lo anterior se traduce como sigue. En preescolar, secundaria y primaria se reconocen tres estadios de la otredad: a) pares escolares (interpersonal), b) el ajeno con cultura propia (histórico-cultural) y c) la vida en comunidad (praxis social) (Teodoro Ramírez, 2007). La otredad en dichos planes de estudio convoca a una posibilidad de respeto, tolerancia y aprendizaje mutuo; siempre, desde un pretendido diálogo y/o conciencia intercultural. En estos ejemplos, los planes de 
estudio fueron investidos como instrumentos que aglutinan un cierto tipo de lucha social y un imaginario colectivo en contra de la discriminación y la exclusión de algunos contextos culturales.

Cuarto, como se hizo notar en la Tabla 2, la noción diversidad se postula como el elemento clave en la configuración del discurso intercultural. En este ensayo se asume que la diversidad cultural es un punto nodal (Laclau y Mouffe, 1987) que atraviesa los marcos figurativos y simbólicos de lo intercultural. Esa diversidad (cultural) se mantiene abierta, susceptible de incorporar distintos sectores poblacionales. Las caracterizaciones que le asignó la SEP fueron variadas (indígenas, jornaleros, migrantes, no indígenas, etc.) y estaban en relación directa con el plan de estudios analizado. De manera metafórica, a este punto nodal -diversidad cultural- se le concibe como un imán que jalonea, atrapa y tensiona "las significaciones que, temporalmente, quedaron bajo su custodia" (Malaga-Villegas, 2012, p. 25) (sic), y que han dado cabida a una reinscripción de lo social para entenderla como hasta ahora se hace. Sin embargo, esto no quiere decir que así haya sido o que así sea siempre.

Al tratarse de un escenario común, la diversidad cultural subyace a la Educación Intercultural. Dicho escenario -diversidad cultural- resultó del momento de articulación entre significantes y significaciones propios de los planes de estudio analizados. Esos significantes se aglutinaron en un espacio de inscripción temático-epistemológico, caótico, de lucha, convivencia y tensión. En la complexión escolarizada que aquí se presentó, los significantes se superpusieron y dominaron el campo de conocimiento de la Educación Intercultural que, a manera de campo de batalla, dieron paso a la emergencia de una doble complexión escolarizada del discurso intercultural (correspondiente a cada plan de estudios y a la suma de ellas en los planes de este siglo). En función a esto, resultó evidente la plena existencia de una deuda histórica de injusticia social a la que han sido sometidos los sectores indígenas $\mathrm{y}$, en general, los excluidos o minorizados.

\section{Reflexiones finales}

La educación intercultural tal y como ha sido concebida por la SEP en los planes de estudio de educación básica aquí analizados, continúa teniendo un sesgo exclusionista. Además, se muestra como perversa, no por el hecho de visibilizar mayormente al sector indígena, sino porque intenta llevar a una igualdad jurídica (de plenitud) a quienes no quieren 
llegar allí, o tal vez no de la manera en que el Estado y la SEP lo asumen. Bajo este supuesto conviene cuestionarse ¿por qué la SEP no ha incorporado a la comunalidad donde así se requiera- u otras propuestas generadas "desde abajo" como vías alternativas (válidas) para pensar la educación-acreditación?

El análisis que se presenta a lo largo de este ensayo, resulta en un triple llamado de atención a los hacedores de políticas educativas de la SEP:

- Urge que profundicen en la revisión de lo producido en el campo de conocimiento de la educación intercultural a nivel nacional. Resulta lamentable que se obvie el horizonte epistemológico de este campo, al que por décadas han contribuido investigadores, especialistas e interesados en el tema.

- Conviene comprender a la diversidad como un recurso de aprendizaje para recrear las prácticas sociales, culturales y educativas de distintas latitudes; y que cada vez menos se vea como un obstáculo o medio de cosificación de ciertas poblaciones del país.

- Apremia una política de educación intercultural seria, que exceda a cualquier transición gubernamental del país, en la que se recuperen las voces y experiencias de los docentes de distintos contextos culturales y geográficos. No basta con una pretendida interculturalización del currículo nacional o con desplazar nociones (educación intercultural por inclusión educativa). Se requieren mecanismos cuidados, en los que esté presente la participación de quienes se dedican a la docencia, para que discutan, revitalicen y replanteen nociones como la aquí revisada.

En este sentido, cada vez son más urgentes este tipo de revisiones críticas, en las que se visibilicen las condiciones de operatividad de determinados conceptos o nociones que se colocan en los planes de estudio, así como sus limitaciones, contradicciones y resistencias (Malaga-Villegas, 2018). Ejemplo de lo anterior son: calidad, competencias, inclusión, evaluación, etc. De lo contrario, se continuará en una lógica reproduccionista de ciertos conceptos, en donde su problematización pase por alto y cuyas significaciones prefiguradas se asuman como verdades incuestionables. 


\section{Referencias}

Aguilar Nery, Jesús. (2004). Hacia una memoria argumental sobre la educación intercultural en México. Una narrativa desde la frontera norte. Revista Mexicana de Investigación Educativa, $\quad$ 9(20), 39-59. Recuperado de http://www.redalyc.org/articulo.oa?id=14002004

Althusser, Louis. (1988). Ideología y aparatos ideológicos del Estado. Freud y Lacan. Buenos Aires, Argentina: Nueva visión.

Amadio, Massimo. (1987). Caracterización de la educación bilingüe intercultural. En Massimo Amadio, Stefano Varese, César Picon. (Comps.), Educación y pueblos indígenas en Centroamérica. Un balance crítico (pp. 19-25). Santiago, Chile: Unesco/OREALC.

Amadio, Massimo, Varese, Stefano y Picon, César (Comps.) (1987). Educación y pueblos indígenas en centroamérica. Un balance crítico. [PDF]. Recuperado de http://unesdoc.unesco.org/images/0007/000732/073236so.pdf

Bascopé Julio, Martín y Caniguan Velarde, Natalia Isabel. (2016). Propuesta pedagógica para la incorporación de conocimientos tradicionales de Ciencias Naturales en Primaria. REDIE. Revista Electrónica de Investigación Educativa, 18(3), 161-175. Recuperado de http://www.redalyc.org/articulo.oa?id=15547471012

Bello Domínguez, Juan. (septiembre, 2009). El inicio de la Educación Bilingüe Bicultural en las regiones indígenas en México. En X Congreso Nacional de Investigación Educativa. Veracruz, México.

Bertely Busquets, María, Dietz, Gunther y Díaz Tepepa, María Guadalupe (Coords.). (2013). Multiculturalismo y educación, Colección de estados de conocimiento. Distrito Federal, México: ANUIES/COMIE.

Bhabha K., Homi. (1996). El entre-medio de la cultura. En Stuart Hall y Pau du Gay (Comps.), Cuestiones de identidad cultural (pp. 94-106). Madrid, España: Amorrortu.

Buenfil Burgos, Rosa Nidia. (2009). Análisis político de discurso e historia de la educación. En Mónica Pini (Comp.), Discurso y educación. Herramientas para el análisis crítico (pp. 77-122). Buenos Aires, Argentina: UNSAM.

Butler, Judith. (2006). Deshacer el género. Barcelona, España: Paidós.

Cano Ruiz, Amanda. (2014). Aprendiendo español en una telesecundaria de contexto popoluca: curriculum-en-acto. CPU-e, Revista de Investigación Educativa, 19, 148-183. Recuperado de http://www.redalyc.org/articulo.oa?id=283131303006

Comboni Salinas, Sonia y Juárez Núñez, José Manuel. (2013). Las interculturalidad-es, identidad-es y el diálogo de saberes. Reencuentro, 66, 10-23. Recuperado de http://www.redalyc.org/articulo.oa?id=34027019002

Derrida, Jacques. (1989). Márgenes de la Filosofía. Madrid, España: Cátedra.

Derrida, Jacques. (1998). Aporías. Barcelona, España: Paidós. 
Diario Oficial de la Federación. (2001). Acuerdo por el que se establece la Coordinación General de Educación Intercultural Bilingüe. Distrito Federal, México.

Diario Oficial de la Federación. (2016). Constitución Política de los Estados Unidos Mexicanos. Distrito Federal, México.

Dietz, Gunther y Mateos Cortés, Laura Selene. (2011). Interculturalidad y educación intercultural en México. Un análisis en su impacto en los modelos educativos mexicanos. Distrito Federal, México: SEP/CGEIB.

Enrique Hamel, Rainer. (2000). Políticas del lenguaje y estrategias culturales en la educación indígena. En Instituto Estatal de Educación Pública de Oaxaca (Ed.), Inclusión y diversidad. Discusiones recientes sobre la educación indígena en México (pp. 130167). Oaxaca, México: IIEPO.

Feltes, Joan Marie y Reese, Leslie. (2014). La implementación de programas de doble inmersión en escuelas multigrados rurales indígenas. Sinéctica, Revista Electrónica de Educación, 43, 1-18. Recuperado de http://www.redalyc.org/articulo.oa?id=99831379002

Foucault, Michel. (1973). El orden del discurso. Barcelona, España: Tusquets.

Fullan, Michael. (2002). Las fuerzas del cambio. Explorando las profundidades de la reforma educativa. Madrid, España: Akal.

Gigante, Elba. (1994). El reto de la pluralidad cultural y étnica a la educación pública, laica, gratuita, democrática y nacional de calidad en México. Distrito Federal, México: UNESCO.

Gigante, Elba, Díaz Couder, Ernesto, Pellicer, Alejandra y Olarte Tiburcio, Eleuterio. (2001). Libros de texto y diversidad cultural. Los libros en lenguas indígenas. Revista Mexicana de Investigación Educativa, 6(12), 1-17. Recuperado de http://www.redalyc.org/articulo.oa?id=14001207

González Apodaca, Érica. (2009). Acerca del multiculturalismo, la educación intercultural y los derechos indígenas en las Américas. CPU-e Revista de Investigación Educativa, 9, 1-11. Recuperado de http://revistas.uv.mx/index.php/cpue/article/view/74/149

Hall, Stuart. (1996) ¿Quién necesita identidad?. En Stuart Hall y Paul du Gay (Comps.), Cuestiones de identidad cultural (pp. 13-39). Madrid, España: Amorrortu.

Kreisel, Maike. (2016). El enfoque intercultural en el currículo de telesecundaria. Elementos para una reflexión crítica. Sinéctica, Revista Electrónica de Educación, 46, 1-18. Recuperado de http://www.redalyc.org/articulo.oa?id=99843455005

Kristeva, Julia. (1978). Semiótica. Madrid: Fundamentos.

Laclau, Ernesto y Mouffe, Chantal. (1987). Hegemonía y estrategia socialista. Hacia una radicalización de la democracia. Madrid, España: FCE. 
Leiva Guerrero, María Verónica. (2014). Reflexiones sobre el rol de la escuela en las relaciones interétnicas, desde la etnopolítica-estatal chilena. Actualidades Investigativas en Educación, 14(3), 1-19. Recuperado de https://revistas.ucr.ac.cr/index.php/aie/article/view/16127/15624

Malaga-Villegas, Sergio Gerardo. (2012). Complexión del discurso intercultural. Una mirada desde la textualidad documental. Pampedia, 9, 22-36. Recuperado de https://www.uv.mx/pampedia/numeros/numero-9/Complexion-del-discursointercultural.pdf

Malaga-Villegas, Sergio Gerardo. (2018). El significante interculturalidad en las políticas de educación básica en México (1994-2006) (Tesis de doctorado inédita). Departamento de Investigaciones Educativas del Cinvestav, Ciudad de México.

Martínez Buenabad, Elizabeth. (noviembre, 2011). La educación indígena e intercultural en México y sus implicaciones en la construcción de ciudadanías. En XI Congreso Nacional de Investigación Educativa. Nuevo León, México.

Mateos Cortés, Laura Selene. (2011). La migración transnacional del discurso intercultural: su incorporación, apropiación y resignificación por actores educativos en Veracruz, México. Quito, Ecuador: Abya Yala.

Mayer, Enrique. (1987). Los alcances de una política de educación bicultural y bilingüe. En Massimo Amadio, Stefano Varese, César Picon. (Comps.), Educación y pueblos indígenas en Centroamérica. Un balance crítico (pp. 27-37). Santiago, Chile: Unesco/OREALC.

Medina Melgarejo, Patricia. (2003). ONG y redes electrónicas en educación intercultural: prácticas y ámbitos en tensión. En María Bertely Busquets (Coord.), La Investigación Educativa en México 1992 - 2002, Volumen 3: Educación, Derechos Sociales y Equidad. Tomo I (pp. 139-164). Distrito Federal, México: COMIE.

Mendoza Zuany, Rosa Guadalupe. (2017). Inclusión educativa por interculturalidad: implicaciones para la educación de la niñez indígena. Perfiles educativos, 39(158), 5269. Recuperado de http://www.scielo.org.mx/pdf/peredu/v39n158/0185-2698-peredu39-158-00052.pdf

Morales Espinoza, María del Coral. (2012). Hacia una comunidad de práctica con enfoque intercultural: la escuela telesecundaria Tetsijtsilin en Tzinacapan, Cuetzalan, Puebla. CPU-e, Revista de Investigación Educativa, 14, 18-43. Recuperado de http://www.redalyc.org/articulo.oa?id=283121840002

Morales Trejo, Carol Graciela. (2015). Orientación educativa e interculturalidad: aportes teórico-prácticos al quehacer profesional en orientación. Actualidades Investigativas en Educación, 15(1), 1-17. doi: https://doi.org/10.15517/aie.v15i1.17626

Organización de las Naciones Unidas para la Educación, la Ciencia y la Cultura. (2000). Marco de acción de Dakar. Educación para todos: cumplir nuestros compromisos comunes. Dakar, Senegal: UNESCO. 
Sartorello, Stefano Claudio. (2014). La Co-teorización intercultural de un modelo curricular en Chiapas, México. Revista Mexicana de Investigación Educativa, 19(60), 73-101. Recuperado de http://www.redalyc.org/articulo.oa?id=14029405005

Secretaría de Educación Pública. (2001). Programa Nacional de Educación 2001-2006. Distrito Federal, México.

Secretaría de Educación Pública. (2004). Programa de Educación Preescolar. Distrito Federal, México.

Secretaría de Educación Pública. (2006). Plan de estudios 2006. Educación Básica. Secundaria. Distrito Federal, México.

Secretaría de Educación Pública. (2007). Programa Sectorial de Educación 2007-2012. Distrito Federal, México.

Secretaría de Educación Pública. (2009). Plan de estudios 2009. Educación Básica. Primaria. Distrito Federal: México.

Secretaría de Educación Pública. (2011). Plan de estudios 2011. Educación Básica. Distrito Federal, México.

Secretaría de Educación Pública. (2013). Programa Sectorial de Educación 2013-2018. Distrito Federal, México.

Secretaría de Educación Pública. (2017). Modelo Educativo para la Educación Obligatoria 2017. Distrito Federal: México.

Teodoro Ramírez, Mario. (2007). Estadios de la otredad en la reflexión filosófica de Luis Villoro. Diánoia, 52(58), 143-175. Recuperado de http://www.scielo.org.mx/pdf/dianoia/v52n58/v52n58a7.pdf

Vaillant, Denise. (2005). Formación de docentes en América Latina. Re-inventando el modelo tradicional. Barcelona, España: Octaedro.

Varese, Stefano, Gigante, Elba, Lewin, Pedro y Pardo, María. (1987). El promotor indígena en el proceso de autoafirmación y desarrollo étnico. En Massimo Amadio, Stefano Varese, César Picon. (Comps.), Educación y pueblos indígenas en Centroamérica. Un balance crítico (pp. 39-75). Santiago, Chile: Unesco/OREALC.

Vezub, Lea F. (2007). La formación y el desarrollo profesional docente frente a los nuevos desafíos de la escolaridad. Profesorado. Revista de currículum y formación del profesorado, 11(1), 1-23. Recuperado de https:/www.ugr.es/ recfpro/rev111ART2.pdf

Zemelman, Hugo y Quintar, Estela. (2007). Conversaciones acerca de interculturalidad y conocimiento. Distrito Federal, México: IPN-Instituto Pensamiento y Cultura en América Latina. 